\title{
PSN: A Prolog declarative model of conceptual knowledge
}

\author{
JACK GREENBAUM and RUSSELL REVLIN \\ University of California, Santa Barbara, California
}

\begin{abstract}
This paper introduces a declarative model of semantic memory, called PSN, written in Prolog. It is shown to be a descendant of Quillian's (1969) Teachable Language Comprehender (TLC) in its structuring of knowledge as a conceptual reticulum and in its use of spreading activation as a retrieval mechanism. PSN goes beyond TLC, however, in its ability to instantiate the essential benchmark properties of human semantic retrieval. As such, it can provide cognitive science with a normative model on which to assess further research and it demonstrates the value of the Prolog language for modeling psychological processes.
\end{abstract}

One of the fundamental problems of artificial intelligence since its inception has been knowledge representation (e.g., Schutzer, 1987). Many of the semantic systems currently in vogue can trace their ancestry to the seminal work of Quillian and his Teachable Language Comprehender (TLC) system (e.g., Quillian, 1968, 1969). TLC offered prescriptions for and a running example of how to build human semantic knowledge and processing into a computer model. The underlying structure of TLC is a network of concepts whose meanings are defined by their links within the structure. Retrieval of facts (i.e., encoding of sentences and answering of questions) is based on a parallel processing mechanism that propagates connections within the network emanating from entry points determined by the query. The system is elegant in its simplicity and has provided impetus for the development of larger and more complex network theories (e.g., Anderson's, 1983, ACT models).

Paradoxically, the potential contributions of TLC may not have been realized because of its early association with cognitive psychology (e.g., Collins \& Quillian, $1969,1970 \mathrm{a}, 1970 \mathrm{~b}, 1970 \mathrm{c}$ ) which may not have fully appreciated TLC's purpose and processing components (see review by Collins \& Loftus, 1975) and which prematurely rejected TLC for failing to describe the questionanswering behavior of college students (e.g., Smith, Shoben, \& Rips, 1974). Our intention here is to redress this treatment of TLC by putting it in a modern frame and recasting it as a declarative system written in Prolog and called Prolog Semantic Network (PSN). This model clarifies the essential elements of Quillian's system, shows that with minimal extensions it can account for the empirical findings of question-answering research, and places the theory squarely within efforts to develop expert systems and to simulate human conceptual process-

Please send requests for reprints or program information to the authors at Department of Psychology, University of California, Santa Barbara, CA 93106 . ing. We proceed in three phases. First, we briefly describe the inherent features of TLC. Second, we offer a set of performative criteria that a sufficient model of human semantic retrieval must meet, in light of which we take up the criticisms of TLC. Finally, we offer a description of PSN and demonstrate how the criteria are met within PSN, as well as how the Prolog environment motivates the structure of PSN.

\section{THE TEACHABLE LANGUAGE COMPREHENDER (TLC)}

TLC stands as an early effort to build a complete system for comprehending natural language (Quillian, 1968). It was designed to comprehend text by relating each assertion of new text to a semantic network representing facts about the world. Admittedly, the system was "developmental": it contained only a small number of examples in its memory and worked only on isolated phrases. TLC resembles current theories (e.g., Anderson, 1983) in its emphasis on two inherent features: knowledge structure and retrieval mechanisms. Knowledge within TLC is construed as a network of relations in which all concepts and their tokens are interrelated as a conceptual reticulum of nodes and labeled links. A concept is represented as a node in the network with attributes of the concept represented as labeled, relational links from the node to other concept nodes. Quillian proposed five different types of links: (1) superordinate/ subordinate (is a), (2) modifier, (3) disjunctive, (4) conjunctive, and (5) a residual class that allows the specification of any relationship that is itself a concept (usually a verb-object relationship). Every concept has a pointer to its immediate superordinate in the network.

\section{Efficient Representation}

Storage of attributes is said to follow a principle of economy: properties of categories are stored nonredundantly at the "highest" node possible. For example, since "ability to fly" is a general property of the category bird, it 
would be stored at the concept node labeled bird, and not redundantly at every instance of the category (e.g., robin). There are at least two versions of "storage economy," confusion among which may have led to early rejections of the TLC proposal (e.g., Conrad, 1972). We take up these interpretations later.

\section{Question-Answering}

Retrieval in TLC is said to have two components: memory search and verification. Memory search is based on a parallel processing mechanism: activation spreads out from a single node to all nodes to which it has pointers (links), and on a second pass to all the units that these units contain pointers to, and so on, for as many such passes as are required to answer a question. The initiating node in this search is specified by the input words. The words might be part of a sentence, such as "Is a robin a bird?"

Search proceeds in two phases, beginning with a quick check that marks the inputs and performs a local search for intersections before branching out to all possible nodes and links. This preliminary analysis considers up to four possible meanings of a word and sees if there is a match. If this preliminary search fails, a broader one is executed.

Verification of the queried relation is determined by an intersection search mechanism. As each node is reached in the propagation of excitation, an activation tag is left that specifies the starting node and the immediate predecessor. When a tag from another starting node is encountered, an intersection between the two nodes has been found. The paths are then evaluated to determine if they satisfy the syntactic and contextual constraints of the query. If a path is rejected, others are considered in the order in which they are found.

\section{BENCHMARKS FOR SEMANTIC RETRIEVAL}

Any theory that is offered as a first approximation to a model of human semantic memory must be evaluated on its ability to account for the basic findings of memory retrieval. TLC and other models have been tested in a largely standardized paradigm. Students are presented with a question (e.g., "Is a canary a bird?"), to which they must answer either "yes" or "no." The time required to answer the question is the basic datum for evaluating the models. There are four regularities that summarize the important findings: (1) semantic distance effect, (2) relatedness effect, (3) priming (or repeated path hypothesis), and (4) processing constraints. We summarize each of these below, before examining the criticisms of TLC and the advantages of PSN.

\section{Semantic Distance Effect}

An immediate implication of the notion of spreading activation is that the further away two nodes are in the network, the longer it will take for an intersecting path to be discovered. This has been consistently reported in the psychological literature (e.g., Collins \& Quillian,
1969). For example, students require less time to answer the superset question "Is a canary a bird?" (where canary and bird are separated by a single is $a$ connection) than "Is a canary an animal?" (where canary and animal are separated by two is a links: canary is $a$ bird is an animal). Similarly for property questions, students require less time to answer "Can a canary sing?" than "Can a canary fly?"' (where can sing is a property connected by a single link with canary, but can fly is a property connected by a single link to bird-hence, two links away from canary).

This latter pair of questions illustrates the concept of cognitive economy, in which properties such as can fly are stored with the "highest" superset possible (in this case, with bird rather than with each appropriate instance of bird, such as canary, robin, etc.), and retrieval first requires activation of these higher nodes. However, this does not preclude the direct storage of properties at the token node.

\section{Relatedness Effect}

The time required to decide whether an instance is a member of its superset varies among instances of the same category. Logically, of course, all instances are equally good members of a category. Yet, definite inequalities in instance-category judgments have been shown to exist at a psychological level. For example, the time to judge that a canary is a bird is less than the time to decide that a goose is a bird (Rips, Shoben, \& Smith, 1973; Smith, Shoben, \& Rips, 1974). Consequently, verification latencies vary with the semantic distance both within and between categories. Specifying the critical features that distinguish among instances of a category has motivated considerable psychological research (e.g., Rips et al., 1973).

\section{Priming (Repeated Path)}

Activated concepts do not instantaneously return to a quiescent state following a response from the conceptual network. The current activation of memory is dependent upon the immediately preceding activation. If two successive questions require the activation of common predicates within conceptual knowledge, then the response to the second question will be facilitated by virtue of having critical paths already activated. For example, the query "Is a canary a bird?' will be responded to by the system more quickly if it has been immediately preceded by a query of one of its components, such as the link between canary and bird that is required to answer the question "Can a canary fly?" than if the immediately preceding query did not reference the same nodes in the network.

\section{Processing Constraints}

It is a common characteristic of question-answering profiles that students are able to retrieve superordinate category information more quickly than superordinate properties. That is, students can answer "Is a canary a bird?" more quickly than "Can a canary fly?" In con- 
trast, this difference between properties and classes does not hold at the immediate level: "Can a canary sing?" is more rapidly answered than "Is a canary a bird?" Any system that seeks to simulate the human questionanswering process must permit this differential accessibility of properties and classes.

\section{LIMITATIONS OF TLC}

The contribution of TLC to our understanding of the question-answering process and natural language comprehension is substantial. It stands historically as the quintessential network system-descendant of list processing efforts and grandparent of today's knowledge/query systems. Its later development, however, was shaped by an effort to provide cognitive psychologists with a normative model of human conceptual knowledge. As such, the diversity of the system was restricted for purposes of exposition and testing. For example, only the quick-check mechanism has been truly examined (e.g., Collins \& Quillian, 1969), and the notion of storage economy is considered only in its strongest form (see discussion by Collins \& Loftus, 1975). In spite of these and other limitations, the psychological version of TLC has been able to capture two of three benchmark regularities mentioned above-namely the semantic distance effect and the repeated path hypothesis (e.g., Collins \& Loftus, 1975; Collins \& Quillian, 1970a, 1970b, 1970c, 1972).

TLC is faulted primarily for its inability to account for "psychological" distance in the network, that is, for the phenomenon of relatedness (Rips, Shoben, \& Smith, 1973; Smith, Rips, \& Shoben, 1974; Smith, Shoben, \& Rips, 1974). Recall that some instances of a category are perceived as "closer" to the superordinate than are other instances. This closeness is negatively correlated with question-answering latencies. TLC is structurally unable to accommodate this finding since all of the links within the network are conceptually the same length. Even with a number of post hoc fixes (e.g., "criteriality") proposed by Collins and Loftus (1975), preserving the hierarchical structure of the system (as it is presented in the psychological literature) makes it incapable of handling "nonlinearities" in the question-answering latencies. For example, students answer the question "Is a dog an animal?" more rapidly than "Is a dog a mammal?" However, in the hierarchical structure of TLC, mammal should be encountered before animal, predicting that the latencies would be the reverse of those observed. We turn now to PSN, which extends TLC and is capable of accommodating all of the benchmark findings.

\section{PSN: PROLOG SEMANTIC NETWORK}

PSN represents knowledge as a declarative network of relations in the spirit of TLC. The model is written in Pro$\log$, which offers a common ground between methods for representing world knowledge and recent advances in the creation of inference engines. ${ }^{1}$ It provides a vehicle for specifying both knowledge and inference rules within a common declarative framework. We view PSN as the basis for a psychological theory of human conceptual knowledge. In its current state, it is able to simulate the benchmark processes that characterize the human question-answering process. It is not intended (as was TLC) as a theory of language comprehension, although it can provide the conceptual underpinnings of such an enterprise. Minimally, PSN can be viewed as a programming strategy as well as a psychological theory.

\section{Knowledge Structure}

A concept in PSN is represented as the predicate,

$$
\text { type(Symbol, [ }<\text { list of properties }>\text { ]). }
$$

For example, PSN's basic knowledge of a canary is given in Listing 1, along with a partial listing of its associated knowledge (e.g., bird, feathers, flying). Notice that the properties associated with canary are themselves concepts with the database. The list of properties is defined in terms of verb-object (vo) predicates that specify a range of relations that are isomorphic to the labeled links in TLCvo("has", "skin'), vo("can", "fly")-as well as superset links (e.g., [superset(bird)]) that define the hierarchical structure of the reticulum.

This structure is similar to TLC in that the list of properties is ordered, starting with superset relations, and then a progression of verb-object relations. We show later, however, that in the present environment, this structure allows PSN to simulate how students access supersets more quickly than properties, as well as how they reorder those properties and classes as a result of the conjoint frequency of queries of the subject-predicate categories.

\section{Search}

In response to a query, such as "Is a canary a bird?" a simple front-end to PSN establishes an initial entry point into the knowledge base by activating the search source, canary. ${ }^{2}$ PSN then evaluates the current knowledge to determine if the target relation is satisfied. This is accomplished by attempting to satisfy the predicate,

$$
\text { search(Subject, Object, Relation), }
$$

where subject and object are variables corresponding to canary and bird, respectively, and relation in the present example refers to "superset" relation. This last argument is required by the kinds of queries that PSN seeks to model. In the typical semantic retrieval task (e.g., Rips, 1975), the student is asked to determine whether the subject and object have a subset-superset relation, and not whether there is a mere connection between them (but see Potts, 1978).

\section{Activation}

A node in PSN is said to be activated when it is marked with an activation tag. These tags hold the name of the 


\section{LISTING 1}

Sample Representation of Knowledge of PSN*

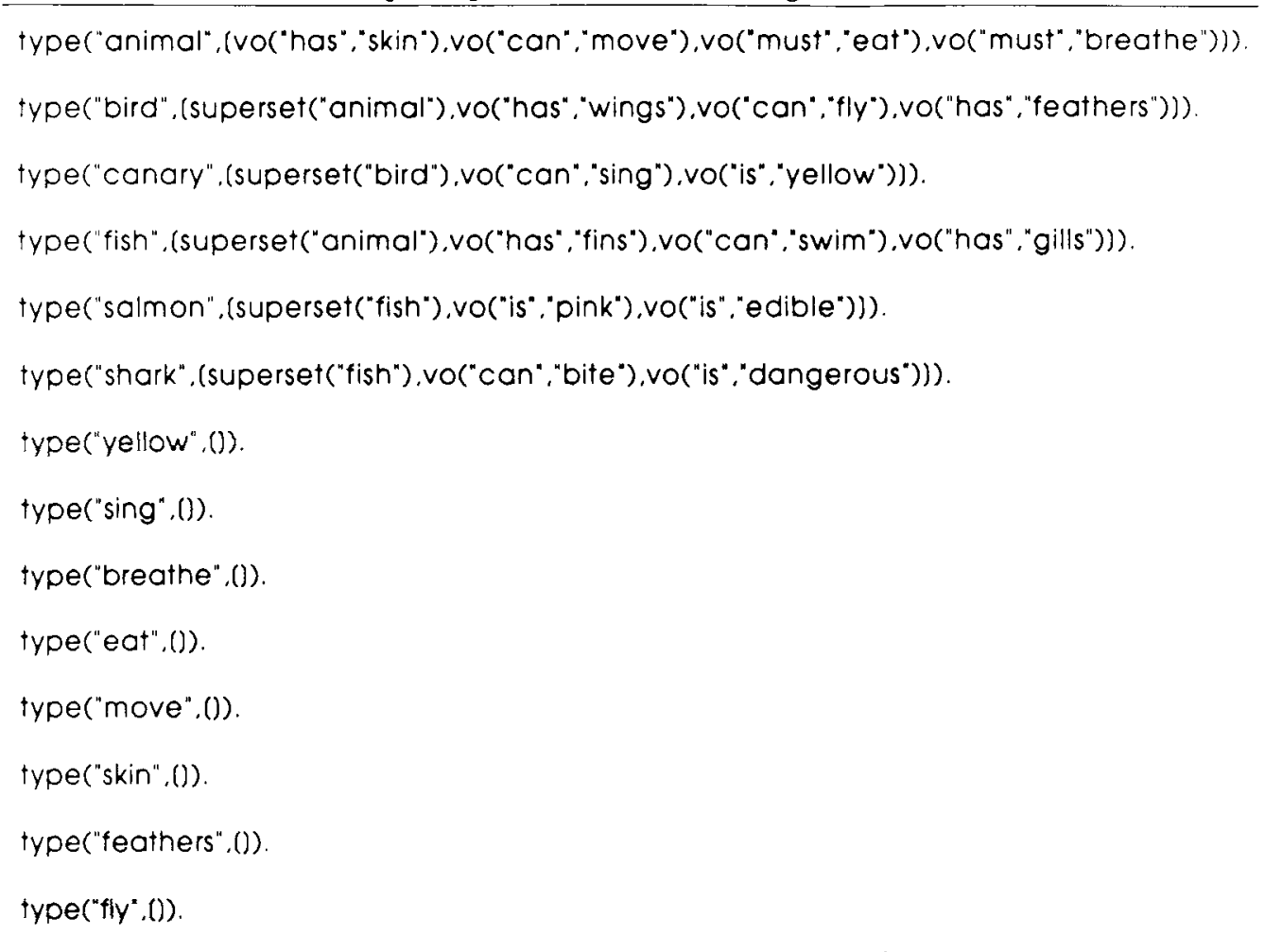

*This is the contents of the database "TLC.dat," which is consulted by PSN.

node from which the search emanates and the last node traversed in that search. The activation tags are expressed by the act() predicate:

$$
\text { act(From, At, Last, Relation). }
$$

The act( ) predicate has four arguments. The first is the search source, followed by the currently activated node. The third corresponds to the current node's immediate predecessor in the search. The fourth argument is the type of relation used to create the activation from the immediate patriarch to the current node.

The termination conditions are checked against the current level of activation. Since all nodes activated on the immediately preceding propagation are already known to not satisfy the search condition, only the new activations need to be checked. PSN expresses these newly activated nodes as the leading_edge( ), which functions as a working memory. Once a leading_edge() has been checked, the information is kept in an activated state as an act() predicate and is replaced by the new leading_edge() composed of its children.

By way of illustration, let us trace PSN as it propagates activation throughout the semantic network in search of verification that a "canary is a bird" (annotated source code of PSN is also provided in the Appendix). To initiate the search, the source, type (canary[...]), is marked to be activated. This is accomplished by asserting the fact,

leading_edge(canary, canary, canary, source).
This means that canary is on the leading edge of spreading activation. The arguments specify that activation began at canary, the immediate patriarch of this activation has been canary, and it has been activated because it is the search source.

The rule, search(), will now attempt to be satisfied. The Prolog clauses involved are illustrated in Listing 2. Search( ) takes the arguments subject, object, and relation, which are bound to canary, bird, and superset, respectively. The repeated propagation of activation throughout the conceptual network terminates when the predicate done( ) is satisfied, or when no more activation can be spread. The predicate done () is satisfied when the

LISTING 2

PSN: The Search Pole

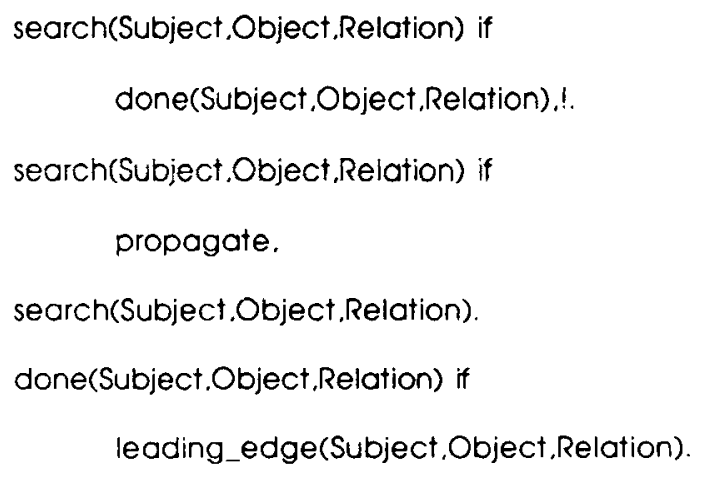


leading_edge() of spreading activation has reached the relationship for which we are searching, that is, when the predicate, leading_edge(canary,bird,_, superset) is true.

In this search to answer "Is a canary a bird?"' done( ) will fail on the first attempt because leading_edge(canary, bird, , , superset) cannot currently be satisfied. PSN will then try the next alternative for search, which propagates activation throughout the next level, and then recurses to see if the search has finished.

In the first pass, leading_edge(canary, canary, canary, source) exists in PSN as the current state of knowledge. As such, it is asserted as activated, and its children are marked as the new leading edge. This is illustrated in Listing 3 , which presents the state of activation at the conclusion of the first pass. As shown by the list of predicates in Listing 3 and in the Appendix, PSN simulates parallel processing by activating all of the properties at a single level. The propagate clause waits for a synchronizing signal before it continues to check the terminating conditions. This is what TLC does with all links emanating from a single node. These newly activated leading_edges are tested recursively within done( ). When leading_edge(canary, bird, canary, superset) is found, search() succeeds. An illustration of the recursive architecture embodied in the goal tree is given in Figure 1.

\section{Effectiveness of PSN}

Semantic distance. The more semantic levels that PSN must examine to answer a question, the greater the overall response latency. This is an inherent feature of all spreading activation models (Anderson, 1983). To determine whether the relative latencies would be equivalent to those obtained in the psychological research, we traced the component processes at each step in the search. PSN simulates the standard distance effect by means of the propagate clause, which is instantiated once for each semantic "link." Since the propagate clause takes a constant amount of time (ceterus paribus), traversing two semantic links will take twice as long as traversing one. This corresponds to the findings of Collins and Quillian (1969).

PSN not only matches the typically shown distance effect, it also accounts for previously unexplained nonlinearities in the latencies. For example, Collins and Quillian (1969) discovered that when students were queried about an identity relation (e.g., "Is a canary a canary?"), their decisions were inordinately fast and deviated from the rec-

\section{LISTING 3}

State of Knowledge Following the First Pass

act(canary, canary, canary, source)

leading_edge(canary,bird,canary,superset)

leading_edge(canary.sing.canary,vo("can","sing"))

leading_edge(canary,yellow,canary.vo("is" , yellow))

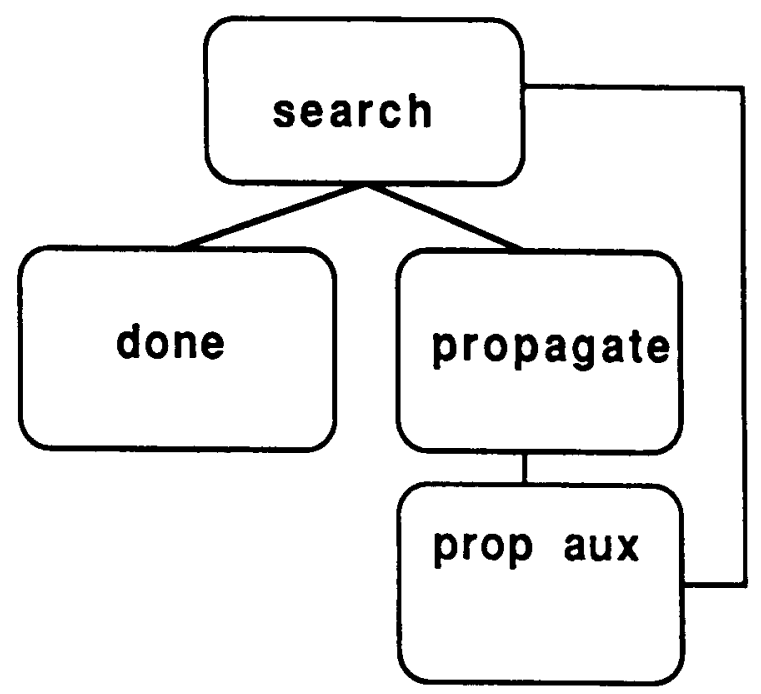

Figure 1. PSN goal tree.

tilinear function that relates decision latency and distance in the network. PSN reproduces this nonlinearity naturally because it always begins the search with an identity predicate and thereby does not entail propagation and activation mechanisms that the more "distant" relations would.

Also reported were different response times for properties versus classes. Although both types of questions showed response time related to semantic distance, property queries took longer. This is expressed within PSN by processing superset/subset and property queries differently. For example, a superset relation may be verified by merely looking at the activation tag, but properties must be searched for at each level of propagation by the done_vo rule (see Appendix). In these ways, PSN accounts for the standard as well as the anomalous findings revealed by Collins and Quillian (1969).

Priming and repeated paths. PSN takes advantage of the way Prolog binds variables, which allows it to keep "activated" the last arguments that constitute the leading edge of activation, as well as any activations expressed as act() facts. When search() is satisfied, the Prolog database remains intact. If another search is initiated, information from the last query is still accessible. For example, if we follow the question "Does a canary have skin?" with "Is a canary an animal?" still on the leading edge of spreading activation from the first question will be the fact

$$
\text { leading_edge(canary, animal, bird, superset). }
$$

This is the activation tag that will satisfy done() during the first call to search(canary, animal, superset), and no propagation of activation need occur. In this way, PSN exhibits the repeated path phenomenon.

TLC placed activation tags at individual nodes in the database. To find an activation, one potentially had to search the entire database. In contrast, PSN embodies 
global activation, in which the activations per se are separate from the total database of knowledge. One consequence of this is to permit repeated queries of the activated materials without having to repeat the entire search process. This facilitates answering repeated queries by means of previously activated nodes.

Eliminating redundant search. Any node may have a multitude of superiors, so that after a few levels of activation in a true network (reticulum) there could be a multitude of activated nodes. If there was no built-in filter to prevent reevaluation of the earliest activations, then the entire activated area of the network (already evaluated) would be repeatedly reevaluated after each spread of activation. PSN efficiently focuses on those activated relations on the leading edge of the spreading activation. To limit redundant evaluations of the same nodes, PSN contains a mechanism that restricts the evaluations to new, potentially relevant connections. This is accomplished during propagation of activation from one semantic level to the next. As each node on the current leading edge activates its children in the progagate rule, the representation of its activation is changed from the leading_edge( ) fact to an act() fact. The newly activated children are now the only leading_edge( ) facts left in the system, and thus are the only activations checked by done( ) for the termination conditions.

Relatedness. A direct consequence of the Prolog architecture is that the binding of variables is determined by the ordering in which the predicates appear in the database, as well as by the ordering of properties within the type node. Since PSN is designed as a first approximation to a simulation of real-world question-answering, the ordering of predicates can be determined by at least two natural constraints: importance and appropriateness. Importance is determined by frequency of use, that is, the conjoint frequency of queries between the subject and predicate terms of the sentence. It can also be affected by the historical sequence in which the relations were learned. Appropriateness is less readily specified. It corresponds to individual intuitions that some predicates should be stored in the vicinity of others or that special predicates should be created that link more elementary ones. This may be occasioned by commonalities noticed by the learner, such as a "family resemblance structure" (Rosch \& Mervis, 1975).

Both importance and appropriateness can affect the ordering of predicates through a mechanism in which the queried predicates "migrate" upward in the ordering. The higher in the ordering, the more quickly they will be bound. In addition, the Prolog environment allows the addition of simple mechanisms that can reorder the property list within each type node to reflect the conjoint frequency of accessing these properties without encumbering the entire data set.

The psychological aspects of relatedness are embodied, then, in two ways within the PSN system: (1) the ordering of predicates in the Prolog database and (2) the ordering of properties on the list of properties within a type node. In this way, PSN can verify quantified queries without direct appeal to the logic of properties and classes, and consequently can avoid an inherent limitation of TLC and similar models, which treat all instances of a class as equivalent (Smith, 1978).

For our first simulation, we used the test data given in Listing 1, taken from Collins and Quillian (1969). The ordering of predicates within the database corresponds to the ordering of importance of those predicates to our test queries. As with TLC, answers to true questions require fewer steps in PSN than do answers to false questions, and questions that require search of distant connections require more steps than those that query less distant relations.

In contrast to TLC, PSN is able to transcend the inherent rigidity of networks by making its database dynamic, by allowing relations to be modified by experience (e.g., importance and appropriateness). A simple reorganization of the list of properties or the ordering of predicates permits a simulation of different category structures and different search sequences. PSN readily allows us to model the development of an optimal conceptual structure or even a "defective" one by adding or deleting clauses. PSN possesses a number of mechanisms that offer increased efficiency over TLC, including the property of globally accessible activation so that the entire network does not have to be searched for previously activated predicates and a leading_edge that highlights the current contents of the knowledge base.

The advantages of PSN cannot be separated from the value of working within the Prolog environment. Prolog's architecture motivated the declarative knowledge structure. Its backtracking features suggested the usefulness of a leading_edge to capture currently activated knowledge, an emergent property of which was the ability to take advantage of repeated paths. In a sense, Pro$\log$ makes the simplicity of PSN possible. We believe that PSN provides a useful example of the simple, declarative framework of Prolog, which makes its models accessible, transparent, and effective.

\section{CONCLUSIONS}

One reason why TLC has achieved such longevity and interest is that it provides a viable framework in which to explore the importance of putative constraints on conceptual systems. It offers cognitive psychologists a normative model in which to address how people are able to store and access conceptual knowledge. The theory was transformed into a psychological processing model by Collins and Quillian (1969) and later substantially modified by Collins and Loftus (1975). Unfortunately, in the process of testing the theory, it has been altered nearly beyond recognition and forced to fit constraints that it was never intended to address. PSN offers a viable alternative model that embodies the inherent features of TLC, but is able to go further by virtue of its Prolog environment. It provides a compact, completely declarative ar- 
chitecture in which to express the essential elements of the human question-answering process as well as a running, normative model to guide further research.

\section{REFERENCES}

Anderson, J. A. (1983). The architecture of cognition. Cambridge, MA: Harvard University Press.

Collins, A. M., \&ofTus, E. F. (1975). A spreading-activation theory of semantic processing. Psychological Review, 6, 407-428.

Coluns, A. M., \& Quluian, M. R. (1969). Retrieval time from semantic memory. Journal of Verbal Learning \& Verbal Behavior, 8, 240-247.

Collins, A. M., Quillian, M. R. (1970a). Does category size affect categorization time? Journal of Verbal Learning \& Verbal Behavior, 9, 432-438.

Collins, A. M., \& Quillian, M. R. (1970b). Experiments on semantic memory and language comprehension. In L. W. Gregg (Ed.), Cognition in learning and memory (pp. 117-147). New York: Wiley.

Collins, A. M., \& Quiluan, M. R. (1970c). Facilitating retrieval from semantic memory: The effect of repeating part of an inference. Acta Psychologica, 33, 304-314.

Collins, A. M., \& Quillian, M. R. (1972). How to make a language user. In E. Tulving \& W. Donaldson (Eds.), Organization of memory (pp. 310-349). New York: Academic Press.

Conrad, C. (1972). Cognitive economy in semantic memory. Journal of Experimental Psychology, 92, 149-154.

PotTs, G. (1978). The role of inference in memory for real and artificial information. In R. Revlin \& R. Mayer (Eds.), Human reasoning (pp. 139-161). Washington, DC: Winston.

QuilliaN, M. R. (1968). Semantic memory. In M. Minsky (Ed.), Semantic information processing (pp. 216-270). Cambridge, MA: MIT Press.
Quillian, M. R. (1969). The Teachable Language Comprehender: A simulation program and theory of language. Communications of the $A C M, 12,459-476$.

RIPS, L. J. (1975). Quantification and semantic memory. Cognitive Psychology, 7, 307-340.

Rips, L. J., Shoben, E. J., \& SMith, E. E. (1973). Semantic distance and the verification of semantic relations. Journal of Verbal Learming \& Verbal Behavior, 12, 1-20.

Rosch, E. H., \& Mervis, C. B. (1975). Family resemblances: Studies in the internal structure of categories. Cognitive Psychology, 7, 573-605.

SCHUTZER, D. (1987). Artificial intelligence: An applications-oriented approach. New York: Van Nostrand Reinhold.

Sмiтh, E. E. (1978). Theories of semantic memory. In W. K. Estes (Ed.), Handbook of learning and cognitive processes (Vol. 6, pp. 156). Hillsdale, NJ: Erlbaum.

Smith, E. E., RIPs, L. J., \& Shoben, E. J. (1974). Semantic memory and psychological semantics. In G. H. Bower (Ed.), The psychology of learning and motivation (Vol. 8, pp. 1-45). New York: Academic Press.

Smith, E. E., Shoben, E. J., \& RIPs, L. J. (1974). Structure and process in semantic memory: A featural model for semantic decisions. Psychological Review, 81, 214-241.

\section{NOTES}

1. We used Borland's Turbo Prolog version 1.1 running under MS-DOS 3.2.

2. For the sake of brevity, this demonstration does not include discussion of how the questions are parsed. We place the initiating conditions of the search source() and leading_edge( ) within the program.

\section{APPENDIX \\ Turbo Prolog Source Code for PSN}

trace

/*This program is a model of conceptual knowledge and question answering using a semantic network data representation. It was written using Borland's Turbo Prolog version 1.1 running under MS-DOS on an PC compatible. The declarations for Turbo Prolog are included below, but may be omitted if using a non-typed version of Prolog. "/

\section{I* start of Turbo declarations "/}

domains

type = superset: superset2; vo(symbol,symbol); source

property = superset(symbol); vo(symbol,symbol)

prop_list $=$ property*

/"Activation relationship types:

superset - direct superset relation from source

superset2 - superset from immediate predecessor only

vo(verb,object) - verb/object relation, i.e. "has feathers"

source - activated as search source

Properties of an object:

superset(symbol) - symbol is a superset of this object

vo(verb,object) - This verb relationship applies to

the object"/

\section{database}

\begin{tabular}{|c|c|}
\hline $\begin{array}{l}\text { type(symbol.prop_list) } \\
\text { leading_edge(symbol,symbol,symbol,type) }\end{array}$ & $\begin{array}{l}\text { /" TLC type node } * \\
/ * \text { place keeper for * }\end{array}$ \\
\hline act(symbol.symbol,symbol,type) & $\begin{array}{l}\text { /" spreading activation "/ } \\
/ \text { base level activation } \%\end{array}$ \\
\hline
\end{tabular}


global predicates

1 " The predicates declared here are assembly language timer routines used to simulate parallel propagation along the leading edge. They were originally written by Byron Sheppard (Byte Magazine, 1/87), and modified or use with Turbo Prolog. "

stoptime(integer, integer, integer) $-(0,0,0)$ language asm startime - language asm

predicates

done(symbol.symbol,type) /" search termination condition "/

done_vo(symbol,symbol.symbol) $/ *$ used for verb/object search */

check_prop(symbol,symbol,prop_list) /* aux clause for done_vo */

search(symbol,symbol,type) $\quad / *$ top level query */

propagate $\quad /$ "spread activation one level */

prop_aux(symbol.symbol,type,prop_list)/" auxiliary clause "/

wait(integer,integer) $\quad / *$ used to simulate parallel processing $*$

demo

/" Demonstrate PSN */

question 1

question2

/" run a query "/

question 3

clauses

I" end of Turbo declarations, beginning of Prolog code "/

/" Instantiate this clause for a demonstration of PSN. By adding the Turbo Prolog trace directive,one can watch the execution of the searches. "\%

demo :-

consult("tlc.dat"). /" tlc.dat contains type nodes from Table 1 "/

question 1 .

question2.

question3.

$/$ " test case for question "is a canary a bird?" "/

questionl :-

asserta(leading_edge(canary,canary,canary,source)).

search (canary,bird superset).

write(" $\backslash n A$ canary is a bird $\backslash n$ ").

/" test case for question "does a canary have feathers?" */

question 2 :-

asserta(leading_edge(canary,canary,canary,source)).

search(canary, feathers, vo(has,feathers)),

write("\nA canary has feathers $\backslash n$ ").

/ test case for question "is a canary an animal?" "/

question 3 :-

asserta(leading_edge(canary,canary.canary.source)),

search (canary,animal,superset).

write(" $\backslash n A$ canary is an animal $\backslash n$ ").

search(Subject, Object,Relation) :done(Subject,Object,Relation),!. / succeed and cut if done */

search(Subject,Object,Relation) :propagate. $\quad /$ " propagate activation one semantic link */ search(Subject,Object,Relation). $\quad /$ see if we are done now */ 


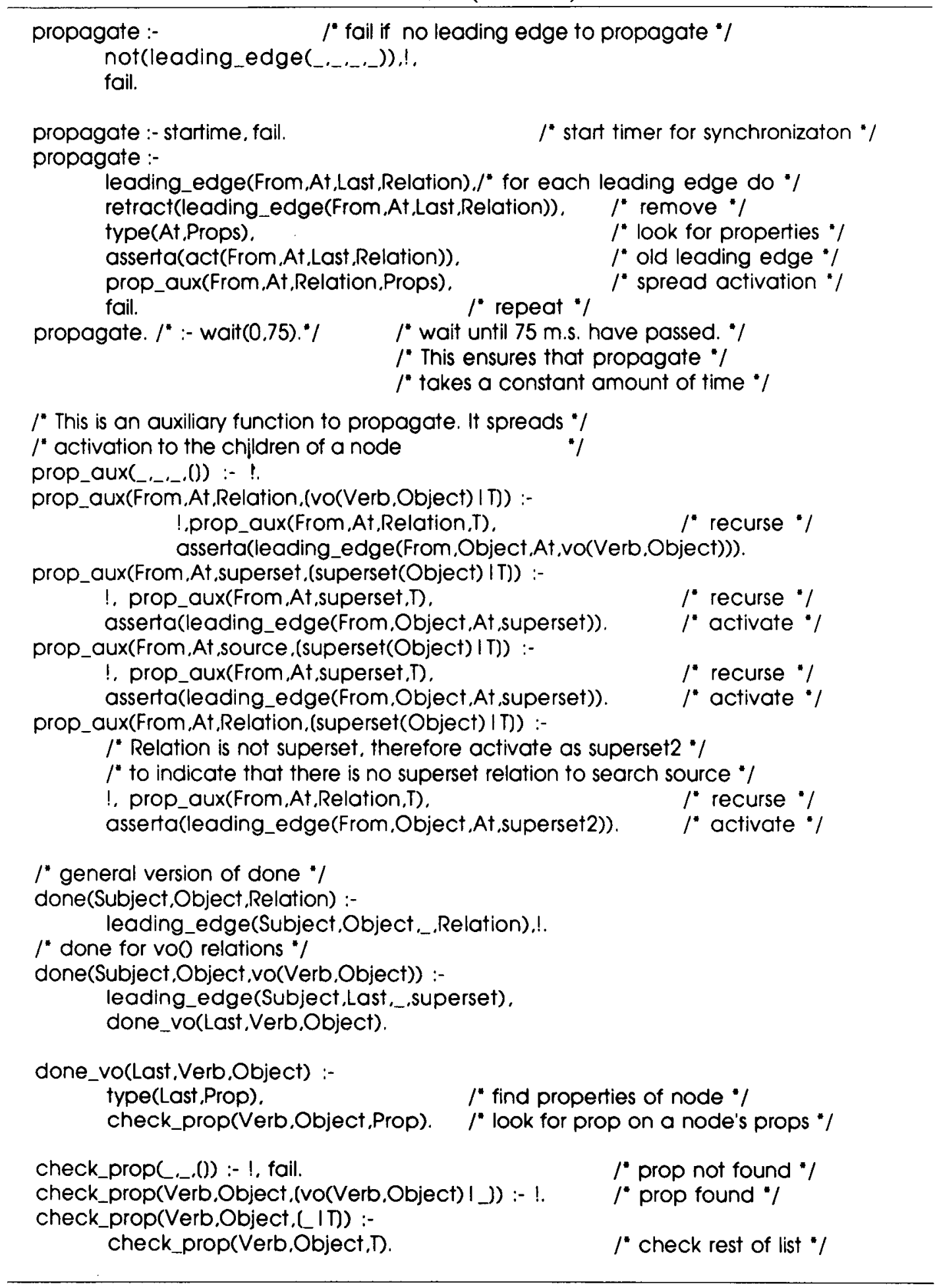

(Manuscript received August 6, 1988;

revision accepted for publication November 29,1988 .) 